\title{
Erratum: Axion global fits with Peccei-Quinn symmetry breaking before inflation using GAMBIT
}

\author{
Sebastian Hoof, ${ }^{a}$ Felix Kahlhoefer, ${ }^{b}$ Pat Scott, ${ }^{a}$ Christoph Weniger ${ }^{c}$ \\ and Martin White ${ }^{d}$ \\ ${ }^{a}$ Department of Physics, Imperial College London, Blackett Laboratory, \\ Prince Consort Road, London SW7 2AZ, U.K. \\ ${ }^{b}$ Institute for Theoretical Particle Physics and Cosmology (TTK), \\ RWTH Aachen University, D-52056 Aachen, Germany \\ ${ }^{c}$ GRAPPA, Institute of Physics, University of Amsterdam, \\ Science Park 904, 1098 XH Amsterdam, Netherlands \\ ${ }^{d}$ Department of Physics, University of Adelaide, \\ Adelaide, SA 5005, Australia \\ E-mail: s.hoof15@imperial.ac.uk, kahlhoefer@physik.rwth-aachen.de, \\ p.scott@imperial.ac.uk, c.weniger@uva.nl, \\ martin.white@adelaide.edu.au
}

ERRATUM TO: JHEP03(2019)191

ARXIV EPRINT: 1810.07192 


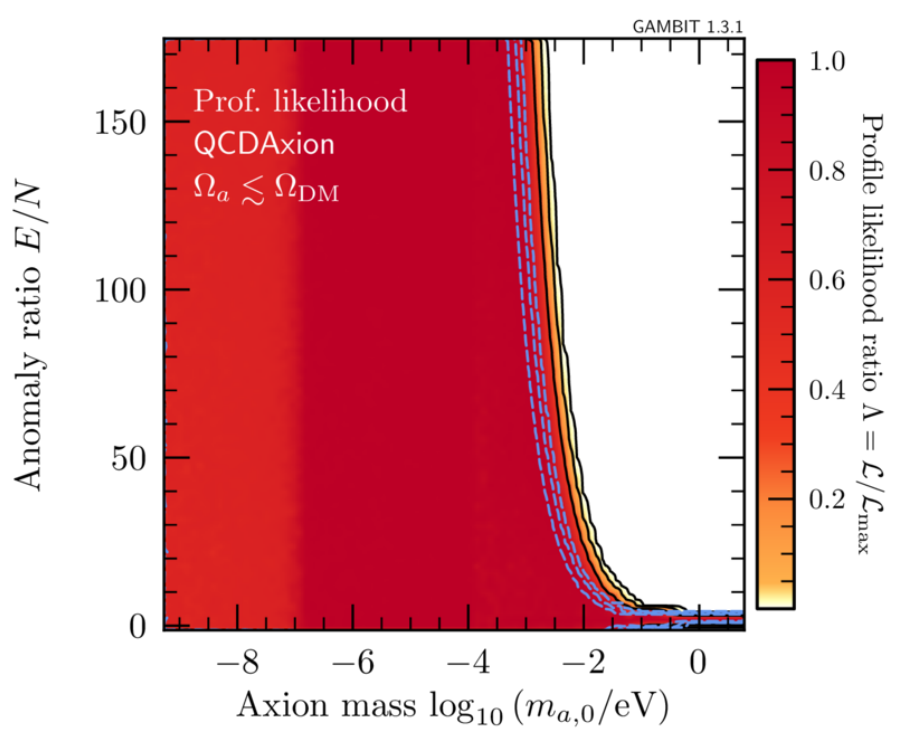

Figure 1. Example for the effect of wrongly defining the axion-photon coupling. The colour map and black lines are for the corrected figure using GAMBIT 1.3.1, while dashed blue lines indicate the previous erroneous exclusion lines from GAMBIT 1.3.0.

We found a bug in the model translation function for $g_{a \gamma \gamma}$ in GAMBIT 1.3.0. The factor of $1 / 2 \pi$ appearing in eq. (3.8) was $1 / \sqrt{2 \pi}$ instead. This affects a number of plots and numerical results for QCDAxion and DFSZ- and KSVZ-type models throughout the paper i.e. both Bayesian and frequentist parameter estimation. The results regarding outcome of model selection are fairly stable against this mistake. The same applies to our qualitative conclusions.

We fixed the bug in GAMBIT 1.3.1 and replaced the samples made available on Zenodo (version v3). We made a completely revised version of the paper available on the ArXiv at https://arxiv.org/abs/1810.07192 (version v3). Here, we therefore only summarise the most notable changes.

In the simplest cases, results are offset by a factor of $\sqrt{2 \pi} \approx 2.51$. We show an example for this in figure 1. Notably, the following figures were affected in a similar fashion: figures 15 (top left), 16 (top left), 19, 20 (left), 21 (top left), 22 (top left), 25 (left), 26, and 27.

- On pg. 39: the upper limit on $m_{a, 0}$ (not demanding that axions are all of the dark matter) changes from $3.8 \mathrm{meV}$ to $7.1 \mathrm{meV}$. The corresponding sentence relating to the DM density should read "The $95 \%$ credibility equal-tailed preferred range is $3.7 \times 10^{-6} \leq \Omega_{a} h^{2} \leq 0.10^{\prime \prime}$, etc.

- On pg. 46: similar to the point above, but when including the cooling hints, the upper limit on $m_{a, 0}$ (not demanding that axions are all of the dark matter) changes 
from $2.8 \mathrm{meV}$ to $6.1 \mathrm{meV}$. The corresponding sentence relating to the DM density should read "At $95 \%$ credibility (equal tails), $5.2 \times 10^{-6} \leq \Omega_{a} h^{2} \leq 0.11$ ", etc.

- On pg. 48, table 8: the column for the best-fit axion masses for KSVZ axions (in meV) should read (from top to bottom): 39.5, 59.4, 316, 105.

Open Access. This article is distributed under the terms of the Creative Commons Attribution License (CC-BY 4.0), which permits any use, distribution and reproduction in any medium, provided the original author(s) and source are credited. 